Review Article

\title{
Adult Patients Affected by Cystic Fibrosis in Therapy with Cystic Fibrosis Transmembrane Regulator Modulators and Lung Transplant: Renal Function, Metabolic and Nutritional Status
}

\author{
Silvia Lai $\left(\mathbb{D},{ }^{1}\right.$ Sandro Mazzaferro, ${ }^{1}$ Anna Paola Mitterhofer, ${ }^{1}$ Francesca Tinti, ${ }^{1}$ Enea Bonci, ${ }^{2}$ \\ Antonello Giovannetti, ${ }^{1}$ Claudio Casella, ${ }^{3}$ Adolfo Marco Perrotta, ${ }^{1}$ Domenico Bagordo, ${ }^{1}$ \\ Manuel Murciano, ${ }_{4}^{4}$ Alessandro Galani, ${ }^{5}$ Camilla Celani, ${ }^{6}$ Patrizia Troiani, \\ Giuseppe Cimino, ${ }^{4}$ and Paolo Palange ${ }^{4}$ \\ ${ }^{1}$ Department of Translational and Precision Medicine, Nephrology and Dialysis Unit, "Sapienza" University of Rome, Rome, Italy \\ ${ }^{2}$ Department of Experimental Medicine, "Sapienza" University of Rome, Rome, Italy \\ ${ }^{3}$ Department of Molecular and Translational Medicine, University of Brescia, Brescia, Italy \\ ${ }^{4}$ Department of Public Health and Infectious Diseases, Special Unit of Cystic Fibrosis, "Sapienza" University of Rome, Rome, Italy \\ ${ }^{5}$ Department of Clinical and Experimental Sciences, University of Brescia, Brescia, Italy \\ ${ }^{6}$ Department of Specialist Paediatric, Rheumatology Unit, Bambino Gesù Children's Hospital, Rome, Italy
}

Correspondence should be addressed to Silvia Lai; silvia.lai@uniroma1.it

Received 19 June 2020; Revised 28 October 2020; Accepted 11 November 2020; Published 30 November 2020

Academic Editor: Jos Mar a Huerta

Copyright (c) 2020 Silvia Lai et al. This is an open access article distributed under the Creative Commons Attribution License, which permits unrestricted use, distribution, and reproduction in any medium, provided the original work is properly cited.

Background. Cystic fibrosis (CF) is one of the most frequent genetic diseases. The discovery and implementation of new therapies prolonged the survival of CF patients in the last years. Evaluation of long-term complications could be useful to improve the outcome of these patients. Aim of the Study. To evaluate renal function, metabolic, nutritional, and inflammatory status in CF patients on cystic fibrosis transmembrane regulator (CFTR) modulators therapy as well as lung transplant recipients (LRs) and patients on conservative therapy (control group). Materials and Methods. We performed a prospective, longitudinal study on 69 CF patients. Clinical and laboratory parameters (metabolic and nutritional indices and inflammatory markers) were evaluated in all patients before starting CFTR therapy or transplant (T0) and after 3 years (T1). Results. We enrolled 69 CF patients (42 males). Patients were distributed into three groups. The average age was $35.01 \pm 10.57$ years for the control group (group 0 ), $32.47 \pm 9.40$ years for patients on CFTR modulators therapy (group 1), and 38.93 \pm 7.14 years for LRs (group 2). At T1, we showed a significant difference among the three groups in terms of renal function indices: creatinine, eGFR, serum nitrogen as well as serum uric acid, sodium, and potassium $(p<0.001, p<0.001, p<0.001, p<0.001, p<0.001$, and $p<0.001$, respectively), particularly in LRs patients. Significant differences were found in nutritional status parameters among the three groups: total protein, serum albumin, serum fibrinogen, serum transferrin, and white blood cell counts $(p<0.001, p=0.037, p=0.04, p=0.003$, and $p=0.007$, respectively), particularly in LRs compared with other groups. Moreover, we found significant differences in metabolic profile (HbA1c, $p=0.026$ ) and inflammatory status, with a significant difference in C-reactive protein values, neutrophil counts, and neutrophil-lymphocyte ratio (NLR) among the three groups $(p<0.001, p=0.005$, and $p=0.026$, respectively). Conclusions. Our study showed a reduced renal function and poor nutritional status in LRs, along with worse metabolic control. Moreover, we showed a lower inflammatory status in patients on CFTR modulators therapy. Therefore, we suggest early and careful monitoring of renal function, metabolic, and nutritional parameters in CF patients, whether they are on conservative therapy, CFTR modulators therapy, and LRs patients. 


\section{Background}

Cystic fibrosis (CF) is the most common autosomal recessive genetic disorder affecting $~ 70,000$ patients worldwide [1]. CF is characterized by chronic airway infection and repeated pulmonary exacerbations which cause lung function decline and poor quality of life, leading to an overall reduction in terms of survival [2]. In these patients, the main cause of respiratory failure is end-stage lung disease, which represents the most common cause of death. Survival rates remarkably improved over the past 40 years, with average survival currently reaching 47.7 years [3]. Advancements in airway clearance, selected antibiotic therapies for chronic infections, and adequate nutrition contributed to ameliorating survival rates [4]. Moreover, new specific treatments were introduced, such as CF transmembrane regulator (CFTR) modulators among others. CF is caused by pathogenic variants of CFTR gene, which is located on chromosome 7 and encodes the CFTR protein, a transmembrane channel of chlorine [5]. This channel is involved in the regulation of anion transport and ciliary mucus clearance in lungs. When these functions are deranged, mucosal retention, chronic pulmonary infections, as well as inflammation occur along the respiratory tract, with a progressive clinical deterioration, which leads to respiratory failure [6]. CFTR modulators represent a targeted treatment, improving the original functions of the defective CFTR protein which causes CF. Modulators are specifically effective on different types or classes of CFTR pathogenic variants carried by the patient. In fact, more than 2000 pathogenic variants of CFTR gene were identified, with the most frequent being $\Delta$ F508 (Class II), characterized by the deletion of three nucleotides which are involved in the coding of phenylalanine [7]. This particular condition causes an altered assembling of the CFTR protein structure, with its consequent degradation by proteasomes in the endoplasmic reticulum, resulting in a lack of exposure of these channels on the cell membrane [4]. All pathogenic variants are categorized into six classes, according to the type of protein deficit [8]. Although such novel therapies considerably improved the outcome of $\mathrm{CF}$ patients, survival rates remain poorer than those observed in general population. In addition, high morbidity affects CF patients' quality of life. In spite of advancements in management and treatment, a large portion of CF patients progresses to end-stage lung disease, finally requiring lung transplant (LT). Lung transplant recipient (LRs) patients' outcome improved over the last 2 decades, with an average posttransplant survival of 8.9 years [9]. Nonetheless, survival rates related to LT are lower than those seen in other solid organ transplantations. In addition, LRs are at high risk for several complications, including acute and chronic rejection, infections, as well as immunosuppressants adverse effects. Therefore, it could be useful to evaluate long-term effects of different therapies along with metabolic, nutritional, and inflammatory status in order to improve CF patients' outcomes.

Aim of the study: to evaluate renal function as well as metabolic, nutritional, and inflammatory status in CF patients treated with CFTR modulators, LRs, and conservative therapy (control group).

\section{Materials and Methods}

The study protocol was approved by the Clinical Research Ethics Committee of Sapienza, University of Rome, Italy. The study conforms to the principles outlined in the Declaration of Helsinki, and we obtained written consent by each patient enrolled.

2.1. Study Design and Subjects. We performed a prospective, longitudinal, single-centre study, with control group, that includes patients aged at least 18 years with diagnosis of CF, afferent to the CF centre at the University Hospital "Policlinico Umberto I" in Rome, Sapienza University of Rome, Italy.

2.2. Patients. A total of 69 patients ( 42 males) were divided into three groups matched by sex and age: patients on conservative therapy (controls) (group 0) (29 patients); patients with CFTR modulators therapy, particularly Orkambi and Kalydeco (group 1) (19 patients); and LRs (group 2) (21 patients). Patients with mutation Classes III and IV (mutations that reduce the function of CFTR proteins at the apical cell surface) can benefit from treatment with potentiators (i.e., VX 770-Ivacaftor, commercialized as Kalydeco ${ }^{\circledR}$ ). Patients with mutation Classes I, II, and V (mutations that reduce the quantity of functional CFTR proteins that reach the apical cell surface) can benefit from treatment with correctors (i.e., VX 809-Lumacaftor, commercialized in combination with Ivacaftor as Orkambi ${ }^{\circledR}$ ) [10]. We performed clinical examinations and blood tests before patients started therapy or underwent LT procedure (T0) and after 3 years (T1), from September 2015 to August 2019. Patients were clinically stable at the time of blood sample, in the absence of exacerbations. We followed the methods of Lai et al. [11].

\subsection{Inclusion Criteria. Patients aged at least 18 years with $\mathrm{CF}$} diagnosis.

2.4. Exclusion Criteria. We excluded patients affected by severe heart disease, ongoing infections, neoplastic disease in progress, as well as chronic liver impairments and cerebrovascular disease. In addition, patients refusing to give consent and those with missing data in their clinical history were excluded.

2.5. Laboratory Measurements. Blood samples were collected in the morning after an overnight fast of at least 12 hours. Standard automated techniques have been used to analyze all patients' samples. We measured the following analytes: plasma glucose (mmol/L), haemoglobin A1c (HbA1c) (\%), total serum cholesterol (mg/dL), triglycerides $(\mathrm{mg} / \mathrm{dL})$, high-density lipoprotein $(\mathrm{mg} / \mathrm{dL})$, creatinine (mg/dL), serum nitrogen $(\mathrm{mg} / \mathrm{dL})$, serum uric acid (SUA) $(\mathrm{mmol} / \mathrm{L})$, calcium $(\mathrm{mg} / \mathrm{dL})$, serum electrolytes $(\mathrm{mEq} / \mathrm{L})$, C-reactive protein $(\mathrm{CRP})(\mu \mathrm{g} / \mathrm{L})$, and total protein $(\mathrm{g} / \mathrm{dL})$. 
Low-density lipoprotein-cholesterol was assessed using the Friedewald equation: low-density lipoprotein (LDL) (mg/ $\mathrm{dL})=$ total cholesterol - high-density lipoprotein-(triglycerides/5). The neutrophil-lymphocyte ratio (NLR) was calculated by dividing the absolute neutrophil counts by the absolute lymphocyte counts. 25Hydroxy-vitamin-D (ng/mL) was measured by radioimmunoassay. Serum albumin $(\mathrm{g} / \mathrm{dL})$ was determined by bromocresol purple method. The estimated glomerular filtration rate (eGFR) was evaluated according to the Modification of Diet in Renal Disease Formula (MDRD), Chronic Kidney Disease-Epidemiology [12].

2.6. Diagnosis of Cystic Fibrosis. Immunoreactive trypsin test in neonatal age was performed. The genetic variants of CFTR were analysed by sequencing analysis and multiple ligationdependent probe amplification (MLPA). Moreover, 17/19 and IR/Del sequencing were performed (INNO-LiPA ${ }^{\circledR}$ CFTR19 (20T)) when necessary. When the found pathogenic variant was not common, molecular investigation of the whole gene was carried out in order to identify the exon deletion [13]. The gold standard for FC diagnosis is the sweat test, in which we perform according to the international guideline. A sweat chlorine concentration greater than $60 \mathrm{mEq} / \mathrm{L}$ supports the diagnosis of CF; an intermediate concentration of chlorine in sweat, between 40 and $60 \mathrm{mEq} /$ $\mathrm{L}$, is suggestive, but not diagnostic, for CF (gray zone); a sweat chlorine concentration of less than $40 \mathrm{mEq} / \mathrm{L}$ is normal and associated with a low probability of CF.

2.7. Blood Pressure Measurements. Blood pressure measurements have been performed using a standard automatic sphygmomanometer according to the British Hypertension Society guidelines [14]. Then, systolic blood pressure (SBP) and diastolic blood pressure (DBP) mean values were calculated for all participants. Hypertension was defined as SBP $\geq 140 \mathrm{mmHg}$ or DBP $\geq 80 \mathrm{mmHg}$ on repeated measurements.

2.8. Statistical Analysis. Data management and analysis were performed using IBM $^{\circledR}{ }^{\circledR}$ SPSS ${ }^{\circledR}$ Statistics 22.0 for Windows ${ }^{\circledR}$ software (IBM Corporation, New Orchard Road Armonk, New York, United States). The Shapiro-Wilk method for normal distributions was used to test the normality of variables. Continuous variables were expressed as average \pm standard deviation; categorical variables were expressed as percentage. The hypothesis testing was performed through the univariate analysis. Following tests were used when appropriate: chi-squared test $\left(\chi^{2}\right)$, Student's $t$-test, analysis of variance (ANOVA), and bivariate correlation (Pearson's $r$ ); values of $p<0.05$ were considered statistically significant.

\section{Results}

A total of 69 patients (42 males) distributed into three groups were evaluated, and the average age was $35.01 \pm 10.57$ years for the control group (group 0), $32.47 \pm 9.40$ years for patients on CFTR modulators therapy (group 1), and $38.93 \pm 7.14$ years for LRs (group 2). We found no significant difference between the 3 groups at T0 (Table 1). Conversely, at $\mathrm{Tl}$, we found significant differences among the three groups with respect to the renal function: creatinine, eGFR, serum nitrogen, SUA, as well as serum sodium and potassium $(p<0.001, p<0.001, p<0.001, p<0.001, p<0.001$, and $p<0.001$, respectively) (Table 2 ), particularly in LRs compared with other groups (Table 3). Regarding the nutritional status, we showed significant differences among the three groups regarding the following analysis: total protein, serum albumin, serum fibrinogen, serum transferrin $(p<0.001, p=0.037, p=0.04$, and $p=0.003$, respectively) (Table 2), particularly in the LRs group (Table 3), although the control group also showed a significant reduction in serum transferrin between T0 and T1 $(p=0.019)$. In the evaluation of metabolic profile, we showed significant difference in the value of HbA1c $(p=0.026)$ (Table 2), with the group of LRs presenting a significant difference between T0 and T1 $(p<0.001)$ (Table 3). With respect to the inflammatory status, we found significant differences in CRP, white blood cells, and NLR among the three groups $(p<0.001$, $p=0.007$, and $p=0.026$, respectively), while neutrophil counts showed a difference in the limits of statistical significance $(p=0.064)$ (Table 2). In particular, we showed a significant increase of CRP value and neutrophil cells in the control group between T0 and T1 ( $p=0.009$ and $p<0.001$, respectively), with a significant reduction of CRP in patients on pharmacological therapy $(p=0.017)$ and a significant increase of neutrophil cells in LRs patients $(p=0.003)$ (Table 3).

\section{Discussion}

Over the last two decades, CF patients' median survival improved, reaching up to 45 years. Improved treatments, such as LT and CFTR modulators, represented a key factor in this challenge $[5,15]$, and therefore, it is now necessary to evaluate the long-term complications of these therapies. In our study, we showed a reduced renal function (with a reduced eGFR) and an increase in serum nitrogen in LRs compared to the other groups of patients. Similarly, Degen et al. [16] and Lai et al. [11] reported a poorer renal function in LRs, probably due to the concurrent administration of immunosuppressive drugs, such as cyclosporine and other calcineurin inhibitors. We found no significant difference in renal function between patients on CFTR modulators and controls. Therefore, the effect of these drugs on CFTRs expressed on the renal tubule seems to be negligible. In fact, although CFTR is widely expressed in kidneys, CF patients do not present major renal dysfunctions. Nevertheless, it is known that both the urinary excretion of proteins and the renal ability to concentrate/dilute urine are altered in these patients [17]. In rats and humans, CFTR mRNA is expressed in all nephron segments, to a greater extent in renal cortex and outer medulla areas. Souza-Menezes et al. [16] showed that CFTR not only has chlorine transport functions but also is probably involved in regulating different ion transport, such as sodium and potassium channels. In kidney, CFTR 
TABle 1: Patients' characteristics at T0.

\begin{tabular}{|c|c|c|c|c|}
\hline T0 & $\begin{array}{c}\text { Group } 0 \\
N=29\end{array}$ & $\begin{array}{c}\text { Group } 1 \\
N=19\end{array}$ & $\begin{array}{c}\text { Group } 2 \\
N=21\end{array}$ & $p$ value \\
\hline Age & $35.01 \pm 10.57$ & $32.47 \pm 9.40$ & $38.93 \pm 7.14$ & 0.092 \\
\hline Creatinine (mg/dL) & $0.75 \pm 0.14$ & $0.76 \pm 0.11$ & $0.83 \pm 0.10$ & 0.060 \\
\hline eGFR (ml/min) & $110.0 \pm 25.26$ & $107.60 \pm 13.98$ & $103.46 \pm 22.42$ & 0.580 \\
\hline Serum nitrogen $(\mathrm{mg} / \mathrm{dL})$ & $5.17 \pm 1.42$ & $5.48 \pm 1.19$ & $5.26 \pm 1.01$ & 0.700 \\
\hline Serum uric acid $(\mathrm{mmol} / \mathrm{L})$ & $0.32 \pm 0.07$ & $0.33 \pm 0.06$ & $0.37 \pm 0.09$ & 0.065 \\
\hline Serum sodium $(\mathrm{mEq} / \mathrm{L})$ & $140.33 \pm 3.23$ & $141.33 \pm 2.11$ & $140.73 \pm 2.32$ & 0.458 \\
\hline Serum potassium $(\mathrm{mEq} / \mathrm{L})$ & $4.56 \pm 0.40$ & $4.43 \pm 0.29$ & $4.58 \pm 0.40$ & 0.387 \\
\hline Total protein $(\mathrm{g} / \mathrm{dL})$ & $7.67 \pm 0.30$ & $7.73 \pm 0.44$ & $7.63 \pm 0.51$ & 0.744 \\
\hline Serum albumin $(\mathrm{g} / \mathrm{dL})$ & $4.51 \pm 0.35$ & $4.46 \pm 0.25$ & $4.42 \pm 0.19$ & 0.552 \\
\hline Serum fibrinogen $(\mathrm{g} / \mathrm{L})$ & $3.31 \pm 0.53$ & $3.49 \pm 0.74$ & $3.33 \pm 0.85$ & 0.658 \\
\hline Serum transferrin $(\mathrm{g} / \mathrm{L})$ & $2.83 \pm 0.48$ & $3.08 \pm 0.60$ & $2.80 \pm 0.37$ & 0.140 \\
\hline White blood cells $\times 10^{3}$ cells $/ \mu \mathrm{L}$ & $8.16 \pm 3.23$ & $7.34 \pm 1.74$ & $7.86 \pm 2.08$ & 0.558 \\
\hline $\mathrm{HbAlc}(\%)$ & $5.53 \pm 0.45$ & $5.86 \pm 1.01$ & $5.38 \pm 0.51$ & 0.074 \\
\hline $\mathrm{CRP}(\mu \mathrm{g} / \mathrm{L})$ & $4663.0 \pm 9948.0$ & $4435.55 \pm 158.26$ & $5400.0 \pm 1096.0$ & 0.883 \\
\hline NLR & $2.39 \pm 1.34$ & $2.37 \pm 1.08$ & $2.15 \pm 0.98$ & 0.750 \\
\hline Neutrophils, $\times 10^{3}$ & $4345.0 \pm 1657.09$ & $4493.0 \pm 1736.34$ & $4696.0 \pm 1740.20$ & 0.773 \\
\hline
\end{tabular}

CRP, C-reactive protein; eGFR, estimated glomerular filtration rate; HbA1c, A1c haemoglobin; N, number of patients; NLR, neutrophil-to-lymphocyte ratio. Data are shown as mean \pm standard deviation or number (\%). Group 0: CF patients on conservative therapy (controls); group 1: CF patients with CFTR modulator therapy; group 2: lung transplant recipient patients.

TABle 2: Patients' characteristics at T1 (after 3 years).

\begin{tabular}{|c|c|c|c|c|}
\hline $\mathrm{T} 1$ & $\begin{array}{c}\text { Group } 0 \\
N=29\end{array}$ & $\begin{array}{c}\text { Group } 1 \\
N=19\end{array}$ & $\begin{array}{c}\text { Group } 2 \\
N=21\end{array}$ & $p$ value \\
\hline Creatinine $(\mathrm{mg} / \mathrm{dL})$ & $0.78 \pm 0.4$ & $0.76 \pm 0.16$ & $1.16 \pm 0.34$ & $p<0.001$ \\
\hline eGFR (ml/min) & $107.66 \pm 25.01$ & $113.0 \pm 22.38$ & $69.38 \pm 20.93$ & $p<0.001$ \\
\hline Serum nitrogen (mg/dL) & $5.21 \pm 1.49$ & $5.52 \pm 1.21$ & $9.64 \pm 3.34$ & $p<0.001$ \\
\hline Serum uric acid $(\mathrm{mmol} / \mathrm{L})$ & $0.32 \pm 0.06$ & $0.34 \pm 0.07$ & $0.43 \pm 0.10$ & $p<0.001$ \\
\hline Serum sodium $(\mathrm{mEq} / \mathrm{L})$ & $141.14 \pm 3.21$ & $140.53 \pm 1.93$ & $143.62 \pm 2.65$ & $p<0.001$ \\
\hline Serum potassium $(\mathrm{mEq} / \mathrm{L})$ & $4.58 \pm 0.32$ & $4.46 \pm 0.27$ & $3.94 \pm 0.52$ & $p<0.001$ \\
\hline Total protein $(\mathrm{g} / \mathrm{dL})$ & $78.52 \pm 6.21$ & $75.58 \pm 5.13$ & $67.29 \pm 4.17$ & $p<0.001$ \\
\hline Serum albumin (g/dL) & $44.55 \pm 4.44$ & $46.26 \pm 4.62$ & $42.74 \pm 3.47$ & $p=0.037$ \\
\hline Serum fibrinogen $(\mathrm{g} / \mathrm{L})$ & $3.52 \pm 0.84$ & $3.17 \pm 0.78$ & $2.93 \pm 0.75$ & $p=0.04$ \\
\hline Serum transferrin $(\mathrm{g} / \mathrm{L})$ & $2.53 \pm 0.36$ & $3.16 \pm 0.64$ & $2.52 \pm 0.61$ & $p=0.003$ \\
\hline White blood cells, $\times 10^{3} / \mu \mathrm{L}$ & $8.58 \pm 2.66$ & $6.50 \pm 1.58$ & $7.85 \pm 1.76$ & $p=0.007$ \\
\hline HbAlc (\%) & $5.74 \pm 0.73$ & $5.98 \pm 0.85$ & $6.54 \pm 1.19$ & $p=0.026$ \\
\hline CRP $(\mu \mathrm{g} / \mathrm{L})$ & $9552.0 \pm 20573.49$ & $1966.67 \pm 2119.75$ & $6265.79 \pm 9016.59$ & $p<0.001$ \\
\hline NLR & $2.89 \pm 4.34$ & $2.44 \pm 0.85$ & $2.70 \pm 1.43$ & $p=0.026$ \\
\hline Neutrophils, $\times 10^{3}(\mu \mathrm{L})$ & $6197.8 \pm 1080.0$ & $5070.52 \pm 1920.0$ & $5897.6 \pm 1900.0$ & $p=0.064$ \\
\hline
\end{tabular}

CRP, C-reactive protein; eGFR, estimated glomerular filtration rate; HbAlc, Alc haemoglobin; N, number of patients; NLR, neutrophil-to-lymphocyte ratio. Data are shown as mean \pm standard deviation or number (\%). Group 0: CF patients on conservative therapy (controls); group 1: CF patients with CFTR modulator therapy; group 2: LT patients.

modulates the expression and controls the activity of renal outer medullary potassium (ROMK) channels, which are at the centre of potassium recycling in the thick ascending loop of Henle and its secretion in cortical collecting ducts [18]. Therefore, CFTR plays a key role in kidneys and, presumably, in other organs where ROMK channels are expressed. Besides, Souza-Menezes et al. [17] described a possible role of CFTR in the epithelial sodium channel $(\mathrm{ENaC})$ regulation, suggesting an interaction between these channels and ROMK channels in renal epithelia where they are coexpressed. In addition to this, the activity of CFTR is influenced by arginine vasopressin, atrial natriuretic peptide, and thyroid hormones, thus indicating the interplay between this channel and the mechanisms of ion transport as well as extracellular volume regulation [19]. However, we found no significant difference in terms of arterial pressure among the three groups of patients. Moreover, CFTR might also be involved in the endocytosis of low-molecular-weight proteins, such as transferrin, in proximal tubule [20]. In fact, in our study, we reported a significant difference in serum transferrin among the three groups. Additionally, as already reported by Lai et al. [11], we found an increase of SUA in LRs with worse renal function. In recent years, many authors showed that high levels of SUA are associated with renal and cardiovascular diseases, mainly due to renal glomerular vasoconstriction [20]. Hyperuricemia is related to endothelial dysfunction and inflammatory and oxidative stress, as the integrity of the endothelium plays a fundamental role in 


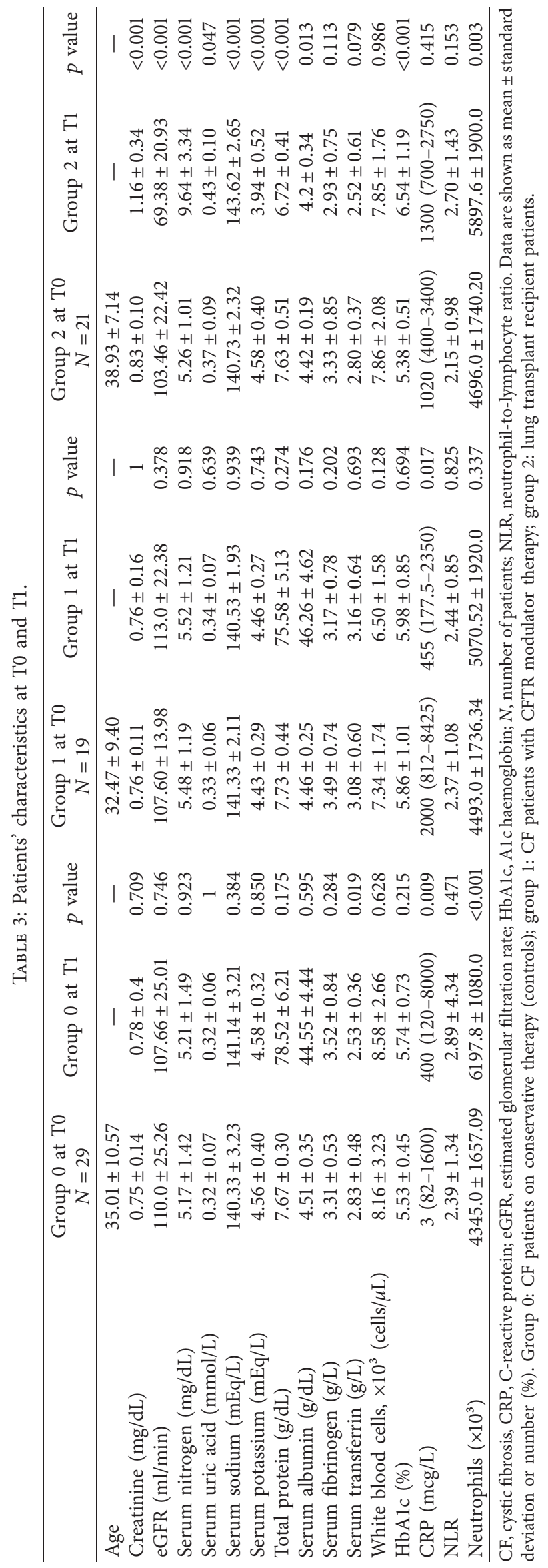


maintaining vascular homeostasis by regulating the balance between vasoconstriction and vasodilation [21, 22]. According to the literature, $40-50 \%$ of CF adult patients are affected by CF-related diabetes (CFRD) [23]. CFRD is the endpoint of a spectrum of glucose abnormalities, which begin with early insulin deficiency and are associated with accelerated nutritional decline and decline of lung function [24]. In our study, we showed significant higher levels of $\mathrm{HbAlc}$ in LRs considered as a marker of worse metabolic balance in this group of patients, not reporting CFRD diagnosis. Indeed, the LRs group showed a poorer nutritional status, as further evidenced by significant differences in total protein, albumin, fibrinogen serum levels, as well as serum transferrin and white blood cell counts. These findings could reflect the greater nutritional vulnerability of LRs [25]. There is a strong association between CF and malnutrition, primarily caused by high energy needs combined with lower nutrient intake. In addition, a well-established correlation between good nutritional status and better lung function was reported. Ashkenazi et al. [26] described for the first time a significant correlation between nutritional status of patients in their infancy and their lung function as adults. Hence, nutritional status should be carefully monitored from early childhood in CF patients and, for this purpose, there is a need for evidence-based recommendations on dietary composition and nutritional guidelines. It is known that body weight disorders, diabetes mellitus (DM), and fatty acid-rich diets produce deleterious effects on immune response, while a "tailored" nutritional approach with accurate evaluation of energy balance remains the cornerstone of $\mathrm{CF}$ therapy since it is associated with a better pulmonary function and prolonged survival [24]. Nonetheless, a significant proportion of patients still fail to achieve normal growth and good nutritional status. In other studies, Colombo [27] and Staufer [28] remarked the importance of nutritional status as an important prognostic factor in patients with CF prior to LT. We found a significant difference in HbAlc among the three groups, with a worse metabolic profile in LRs. This is probably due to the role played by immunosuppressants in both renal function decline and metabolic profile derangement, leading to a greater cardiovascular risk. Similarly, Winhofer et al. [29] reported profound alterations in glucose metabolism, with a high rate of undetected DM and markedly delayed insulin secretion in LRs. Postprandial hyperglycaemia is mainly caused by the impaired beta-cell glucose sensitivity rather than insulin resistance and is accompanied by disturbances in lipid metabolism. Nevertheless, Valour et al. [30] and Hofer et al. [31] did not see a worsening in CFRD and in the survival of LRs with DM. In fact, even though LT is known to impair glucose tolerance, evolution of pre-existing DM is not described in transplanted CF patients. For this reason, although the association with more severe phenotypes in CF patients, it is unclear whether DM influences the LT outcome. With respect to CFRD, a strong relation with lung function decline and increased mortality was reported [24]. Martín-Frías et al. [32] showed that good CFRD metabolic control and adequate nutrition have prolonged beneficial effects on LRs. With regards to inflammatory status, we found a significant difference in CRP levels among the three groups, with a significant increase in patients receiving conservative therapy and a significant reduction in patients on CFTR modulators therapy. Li et al. [33] reported a role of CFTR in preventing inflammation and atherogenesis via inhibition of both $\mathrm{NF} \kappa \mathrm{B}$ and MAPKs activation, therefore suggesting that CFTR might be a potential therapeutic target for the treatment of vascular inflammation as well as the development of atherosclerotic disease. Furthermore, CFTR dysfunction was shown to result in proinflammatory responses in CF patients, but its role in vascular inflammation and atherogenesis remains unclear. In our study, also neutrophil cells count was increased in the control group and in LRs patients. This finding could be explained by the effects of CFTR modulators therapy, suggesting that acting pharmacologically on this receptor might reduce the inflammatory response. High neutrophil levels are associated with CF, and delayed neutrophil apoptosis is also described in this disease, albeit it is unclear whether this condition is due to a primary neutrophil defect or chronic inflammation. Gray et al. [34] showed how CF patients present neutrophils with prosurvival phenotype, associated with absent CFTR function and increased neutrophil extracellular traps production, which might induce inflammation. In our study, we found a significant increase in neutrophil counts in CF patients not receiving receptor modulating therapy and in LRs patients, while NLR was significantly different between the 3 groups at $\mathrm{Tl}$, being greater in the control group but without a significant difference between $\mathrm{T} 0$ and $\mathrm{T} 1$. Nacaroglu et al. [35] reported that leukocyte and platelet count as well as absolute neutrophil count and NLR might be helpful to assess chronic inflammation, but only NLR and absolute neutrophil count should be used as biomarkers of acute exacerbations, an exclusion criterion in our study. Similarly, O'Brien et al. [36] reported a significant correlation between NLR and clinical conditions in child patients, suggesting that NLR might be a potentially useful index of clinical evaluation in CF.

\section{Limitations}

The limitations of our study are the limited sample size of CF patients, and the single-centre study. The limitation for all single-centre analyses is the potential lack of generalizability. Additional prospective follow-up studies with a larger number of patients are needed to confirm our results.

\section{Conclusions}

CF outcomes and survival improved over the last years, mainly due to the advancements in specific therapies and LT-improved management [9]. CFTR modulators have been developed to correct the underlying pathogenetic mechanism of CF, although they are not eligible for all classes of mutation. Moreover, there is a need to evaluate extrapulmonary long-term complications, such as metabolic, nutritional, and inflammatory status. Our study reports a reduced renal function and poorer nutritional status in LRs, along with worse metabolic control. In addition, we showed 
a reduced general phlogosis in patients on CFTR modulators therapy. Therefore, we suggest an early and careful monitoring of renal function and metabolic and nutritional indices in CF patients, whether these patients are on conservative therapy, CFTR modulators therapy, or after LT.

\section{Abbreviations}

CF: $\quad$ Cystic fibrosis

CFRD: CF-related diabetes

CFTR: Cystic fibrosis transmembrane regulator

CRP: C-reactive protein

DM: Diabetes mellitus

DBP: Diastolic blood pressure

eGFR: Estimated glomerular filtration rate

ENaC: Epithelial sodium channel

HbA1c: Haemoglobin A1c

LDL: Low-density lipoproteins

MDRD: Modification of Diet in Renal Disease Formula

ROMK: Renal outer medullary potassium

SUA: $\quad$ Serum uric acid

LT: $\quad$ Lung transplant

LRs: $\quad$ Lung transplant recipients

SBP: $\quad$ Systolic blood pressure.

\section{Data Availability}

The datasets generated during and/or analysed during the current study are available from the corresponding author on reasonable request.

\section{Ethical Approval}

The study protocol was approved by the Local Clinical Research Ethics Committee, Sapienza University of Rome. The study conforms to the principles outlined in the Declaration of Helsinki.

\section{Consent}

We obtained a written consent by each patient enrolled.

\section{Conflicts of Interest}

The authors declare no conflicts of interest.

\section{Authors' Contributions}

SL, SM, APM, GC, and PP contributed to conception and design and interpretation of data; PGM, FP, and MM acquired the data; and CC, PT, and EB analyzed the data. All authors participated in drafting the article or revising it critically for important intellectual content. The authors are responsible for the content and writing of the paper. All authors gave final approval of the version to be submitted.

\section{References}

[1] J. Stuart Elborn, "Cystic fibrosis," Lancet, vol. 388, no. 10059, pp. 2519-2531, 2016.
[2] A. D. Jackson and C. H. Goss, "Epidemiology of CF: how registries can be used to advance our understanding of the $\mathrm{CF}$ population," Journal of Cystic Fibrosis, vol. 17, no. 3, pp. 297-305, 2018.

[3] C. Castellani and B. M. Assael, "Cystic fibrosis: a clinical view," Cellular and Molecular Life Sciences, vol. 74, no. 1, pp. 129-140, 2016.

[4] B. W. Ramsey, M. S. Pepe, J. M. Quan et al., "Intermittent administration of inhaled tobramycin in patients with cystic fibrosis," New England Journal of Medicine, vol. 340, no. 1, pp. 23-30, 1999.

[5] E. B. Burgener and R. B. Moss, "Cystic fibrosis transmembrane conductance regulator modulators," Current Opinion in Pediatrics, vol. 30, no. 3, pp. 372-377, 2018.

[6] M. J. Welsh and A. E. Smith, "Molecular mechanisms of CFTR chloride channel dysfunction in cystic fibrosis," Cell, vol. 73, no. 7, pp. 1251-1254, 1993.

[7] M. Gentzsch and M. A. Mall, "Ion channel modulators in cystic fibrosis," Chest, vol. 154, no. 2, pp. 383-393, 2018.

[8] J. P. Lynch, D. M. Sayah, J. A. Belperio, and S. S. Weigt, "Lung transplantation for cystic fibrosis: results, indications, complications, and controversies," Seminars in Respiratory and Critical Care Medicine, vol. 36, no. 2, pp. 299-320, 2015.

[9] N. E. West and P. A. Flume, "Unmet needs in cystic fibrosis: the next steps in improving outcomes," Expert Review of Respiratory Medicine, vol. 12, no. 7, pp. 585-593, 2018.

[10] K. MacDonald, "Cystic fibrosis transmembrane regulator protein mutations: "class" opportunity for novel drug innovation," Paediatric Drugs, vol. 9, pp. 1-10, 2007.

[11] S. Lai, S. Mazzaferro, A. P. Mitterhofer et al., "Renal involvement and metabolic alterations in adults patients affected by cystic fibrosis," Journal of Translational Medicine, vol. 17, no. 1, p. 388, 2019.

[12] A. S. Levey, J. Coresh, T. Greene et al., "Using standardized serum creatinine values in the modification of diet in renal disease study equation for estimating glomerular filtration rate," Annals of Internal Medicine, vol. 145, no. 4, pp. 247-254, 2006.

[13] P. R. Sosnay, T. B. White, P. M. Farrell et al., "Diagnosis of cystic fibrosis in nonscreened populations," The Journal of Pediatrics, vol. 181, pp. S52-S57, 2017.

[14] C. Antza, I. Doundoulakis, S. Stabouli, and V. Kotsis, "Comparison among recommendations for the management of arterial hypertension issued by last US, Canadian, British and European guidelines," High Blood Pressure \& Cardiovascular Prevention, vol. 25, no. 1, pp. 9-16, 2018.

[15] D. Salvatore, V. Terlizzi, M. Francalanci et al., "Ivacaftor improves lung disease in patients with advanced CF carrying CFTR mutations that confer residual function," Respiratory Medicine, vol. 171, Article ID 106073, 2020.

[16] D. A. Degen, J. Janardan, K. A. Barraclough et al., "Predictive performance of different kidney function estimation equations in lung transplant patients," Clinical Biochemistry, vol. 50, no. 7, pp. 385-393, 2017.

[17] J. Souza-Menezes and M. M. Morales, "CFTR structure and function: is there a role in the kidney?" Biophysical Reviews, vol. 1, no. 1, pp. 3-12, 2009.

[18] W. Wang, S. C. Hebert, and G. Giebisch, "Renal K+ channels: structure and function," Annual Review of Physiology, vol. 59, no. 1, pp. 413-436, 1997.

[19] F. Amato, P. Scudieri, I. Musante et al., "Two CFTR mutations within codon 970 differently impact on the chloride channel functionality," Human Mutation, vol. 40, no. 6, pp. 742-748, 2019.

[20] F. Jouret, A. Bernard, C. Hermans et al., "Cystic fibrosis is associated with a defect in apical receptor-mediated endocytosis in 
mouse and human kidney," Journal of the American Society of Nephrology, vol. 18, no. 3, pp. 707-718, 2007.

[21] F. Viazzi, B. Bonino, E. Ratto, G. Desideri, and R. Pontremoli, "Iperuricemia e rischio renale," Giornale Italiano di Nefrologia, vol. 32, 2015.

[22] T. Maruhashi, I. Hisatome, Y. Kihara, and Y. Higashi, "Hyperuricemia and endothelial function: from molecular background to clinical perspectives," Atherosclerosis, vol. 278, pp. 226-231, 2018.

[23] S. Lai, A. Mariotti, B. Coppola et al., "Uricemia and homocysteinemia: nontraditional risk factors in the early stages of chronic kidney disease-preliminary data," European review for medical and pharmacological sciences, vol. 18, no. 7, pp. 1010-1017, 2014.

[24] S. Lanng, B. Thorsteinsson, J. Nerup, and C. Koch, "Influence of the development of diabetes mellitus on clinical status in patients with cystic fibrosis," European Journal of Pediatrics, vol. 151, no. 9, pp. 684-687, 1992.

[25] V. Boudreau, Q. Reynaud, C. L. Dubois et al., "Screening for cystic fibrosis-related diabetes: matching pathophysiology and addressing current challenges," Canadian Journal of Diabetes, vol. 40, no. 5, pp. 466-470, 2016.

[26] M. Ashkenazi, N. Nathan, I. Sarouk et al., "Nutritional status in childhood as a prognostic factor in patients with cystic fibrosis," Lung, vol. 197, no. 3, pp. 371-376, 2019.

[27] C. Colombo, R. M. Nobili, and G. Alicandro, "Challenges with optimizing nutrition in cystic fibrosis," Expert Review of Respiratory Medicine, vol. 13, no. 6, pp. 533-544, 2019.

[28] K. Staufer, E. Halilbasic, P. Hillebrand et al., "Impact of nutritional status on pulmonary function after lung transplantation for cystic fibrosis," United European Gastroenterology Journal, vol. 6, no. 7, pp. 1049-1055, 2018.

[29] Y. Winhofer, P. Wolf, P. Fellinger et al., "Markedly delayed insulin secretion and a high rate of undetected overt diabetes characterize glucose metabolism in adult patients with cystic fibrosis after lung transplantation," Endocrine Practice, vol. 25, no. 3, pp. 254-262, 2019.

[30] F. Valour, C. Brault, F. Abbas-Chorfa et al., "Outcome of cystic fibrosis-related diabetes two years after lung transplantation," Respiration, vol. 86, no. 1, pp. 32-38, 2013.

[31] M. Hofer, C. Schmid, C. Benden et al., "Diabetes mellitus and survival in cystic fibrosis patients after lung transplantation," Journal of Cystic Fibrosis, vol. 11, no. 2, pp. 131-136, 2012.

[32] M. Martín-Frías, L. Máiz, A. Carcavilla, and R. Barrio, "Efecto beneficioso y prolongado del buen control metabólico de la diabetes relacionada con fibrosis quística sobre la función pulmonar y el estado nutricional," Archivos de Bronconeumología, vol. 47, no. 10, pp. 531-534, 2011.

[33] Z. Li, Z. Shen, H Xue et al., "CFTR protects against vascular inflammation and atherogenesis in apolipoprotein E-deficient mice," Bioscience Reports, vol. 37, no. 4, Article ID BSR20170680, 2017.

[34] R. D. Gray, G. Hardisty, K. H. Regan et al., "Delayed neutrophil apoptosis enhances NET formation in cystic fibrosis," Thorax, vol. 73, no. 2, pp. 134-144, 2018.

[35] H. T. Nacaroglu, S. B. Erdem, S. Karaman, S. Yazıcı, and D. Can, "Can mean platelet volume and neutrophil-to-lymphocyte ratio be biomarkers of acute exacerbation of bronchiectasis in children?" Central European Journal of Immunology, vol. 42, no. 4, pp. 358-362, 2017.

[36] C. E. O’Brien and E. T. Price, "The blood neutrophil to lymphocyte ratio correlates with clinical status in children with cystic fibrosis: a retrospective study," PLoS One, vol. 8, no. 10, Article ID e77420, 2013. 